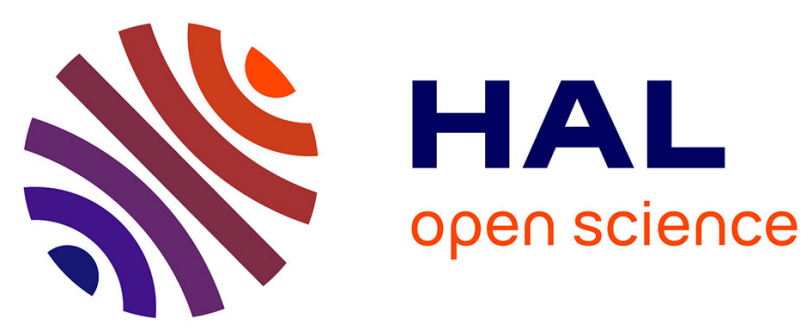

\title{
ANALYSE DES MÉCANISMES D'ENDOMMAGEMENT D'UNE PLAQUE COMPOSITE IMPACTÉE
}

F. Chaillou, C. Cazeneuve, J. Lataillade

\section{- To cite this version:}

F. Chaillou, C. Cazeneuve, J. Lataillade. ANALYSE DES MÉCANISMES D'ENDOMMAGEMENT D'UNE PLAQUE COMPOSITE IMPACTÉE. Journal de Physique IV Proceedings, 1991, 01 (C3), pp.C3-43-C3-50. 10.1051/jp4:1991306 . jpa-00249897

\section{HAL Id: jpa-00249897 https://hal.science/jpa-00249897}

Submitted on 1 Jan 1991

HAL is a multi-disciplinary open access archive for the deposit and dissemination of scientific research documents, whether they are published or not. The documents may come from teaching and research institutions in France or abroad, or from public or private research centers.
L'archive ouverte pluridisciplinaire HAL, est destinée au dépôt et à la diffusion de documents scientifiques de niveau recherche, publiés ou non, émanant des établissements d'enseignement et de recherche français ou étrangers, des laboratoires publics ou privés. 


\title{
ANALYSE DES MECANISMES D'ENDOMMAGEMENT D'UNE PLAQUE COMPOSITE IMPACTÉE
}

\author{
F. CHAILLOU* ${ }^{*}$ C. CAZENEUVE ${ }^{*}$ et J.L. LATAILLADE* * \\ *ETCA/CREA, 16 bis, avenue Prieur de la côte d'Or, F-94114 \\ Arceuil, France \\ **École Nationale Supérieure des Arts et Métiers, F-33400 \\ Talence, France, et, UA CNRS 867, Laboratoire de Mécanique \\ Physique, Université de Bordeaux I, F-33405 Talence, France
}

Résumé - Dans ce travail, on s'intéresse au comportement de plaques composites à matrice organique (thermodurcissable ou thermoplastique) et à fibres longues impactées par des éclats shériques de faible masse (bille de 1 gramme, de $6 \mathrm{~mm}$ ), et de vitesse moyenne comprise entre 100 et $300 \mathrm{~m} / \mathrm{s}$. Une modélisation analytique permet, à partir d'un bilan énergétique, de calculer l'énergie consommée par délaminage. Les modes et la vitesse de propagation du délaminage sont ensuite étudiés par simulation numérique et analytique.

\begin{abstract}
This paper describes the behaviour of fibers reinforced plastic plates impacted by kinetics splinters (sphere of $1 \mathrm{~g}$ and diameter equal to. $6 \mathrm{~mm}$ ), and of medium velocity include between $100 \mathrm{~m} / \mathrm{s}$ and $300 \mathrm{~m} / \mathrm{s}$. The energy absorbed by the delamination was calculated by an analytical model. The modes and the velocity of propagation of the delamination were investigated by a numerical and analytical method.
\end{abstract}

\section{I - INTRODUCTION}

Les structures composites, qu'elles soient destinées aux secteurs aéronautique, automobile ou maritime peuvent être soumises à des chocs de grêlons, de graviers ou d'éclats résultants d'explosion. C'est pourquoi, dans cette étude, nous allons nous intéresser à l'impact de projectiles de quelques grammes sur des plaques composites à fibres longues et à matrice organique dont les vitesses incidentes sont comprises entre 100 et $300 \mathrm{~m} / \mathrm{s}$.

Les modes de ruine de la plaque varient selon ses caractéristiques et celles du projectile. DOREY [1] en discerne trois : - la rupture en délaminage ( fig. 1a )

- la rupture en flexion ( fig. $1 \mathrm{~b}$ )

- la rupture par poinçonnement et création d'un bouchon à vitesse élevée ( fig. 1c )

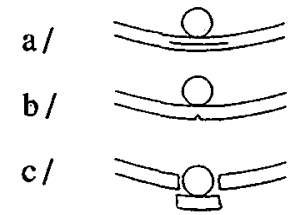

FIGURE 1 - Modes de ruine à l'amorçage d'après Dorey (1982)

Le plus souvent, dans la gamme de vitesses étudiées, on constate un endommagement par délaminage. Celui-ci apparaît aux interfaces présentant le plus grand changement d'orientation [2-3]. De plus, les caractéristiques mécaniques d'une structure délaminée chutent fortement [4].

C'est pourquoi, nous allons nous intéresser à la propagation du délaminage lors d'un impact.

L'amorçage du délaminage sous impact peut être abordé par calcul par éléments finis [5] : le champ de contraintes à chaque instant est calculé, puis un critère d'initiation permet de déterminer l'instant d'amorçage. 
La propagation du délaminage peut être étudiée expérimentalement et théoriquement. Une première méthode consiste à créer, dans l'éprouvette, un défaut dont on observera la propagation lors de l'impact. L'éprouvette peut être une poutre [6-9] dont la fissure est suivie au cours du temps par une caméra ultrarapide. Les calculs par éléments finis permettent de déterminer le taux de restitution d'énergie en fonction du temps.

Certains auteurs [10] considèrent des plaques avec des défauts circulaires. La surface délaminée autour du défaut est contrôlée, après chargement, par ultra-sons.

Une autre méthode consiste à établir un modèle numérique [11-12] ou analytique [13] permettant de déterminer la surface délaminée. Cette méthode est ensuite validée par confrontation avec l'expérience.

Dans cette étude, nous nous proposons de comprendre comment le délaminage se propage lors de l'impact. Pour cela, nous allons d'abord calculer globalement l'énergie consommée par le délaminage lors de sa propagation. Ensuite, nous déterminerons le mode de propagation du délaminage puis sa vitesse d'extension dans le plan de la plaque.

\section{II - DISPOSITIF EXPERIMENTAL}

Les tests d'impact sont réalisés à l'aide d'un canon à air comprimé, les vitesses incidentes et résiduelles (après perforation) sont mesurées par un système optique et la visualisation des phénomènes est obtenue par une caméra ultra-rapide. Celle-ci permet d'obtenir huit à dix clichés en temps réel, espacés de une à cent micro-secondes.

Les matériaux testés sont des composites à fibres de carbone ou de verre et à matrice thermoplastique (PEEK) ou thermodurcissable (5208 - 5245C).

\section{III - PHENOMENOLOGIE}

L'impact d'un projectile sur une plaque composite va créer un dôme de flexion. Celui-ci a une base circulaire dans le cas d'un empilement quasi-isotrope ou elliptique dans le cas d'un empilement plus anisotrope : c'est ce que l'on appelle le relèvement de la plaque (figure 2).
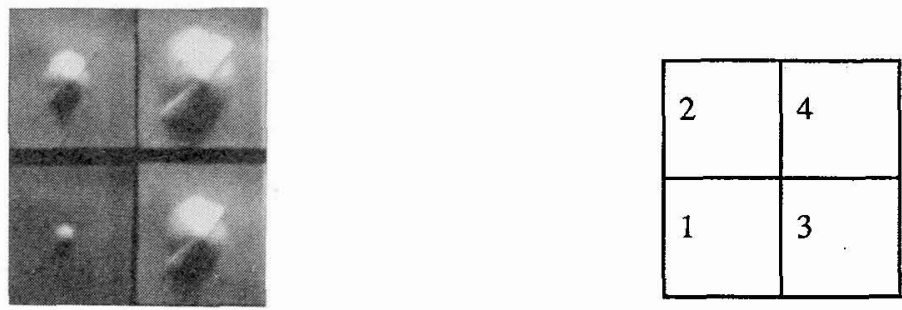

FIGURE 2 : Face arrière impactée - 1 image par $20 \mu \mathrm{s}$ - V incidente : $227 \mathrm{~m} / \mathrm{s}$

La vitesse de relèvement Ur est la vitesse d'extension de la base du dôme. Le point de relèvement Ar est le point virtuel qui se trouve à la base du dôme.

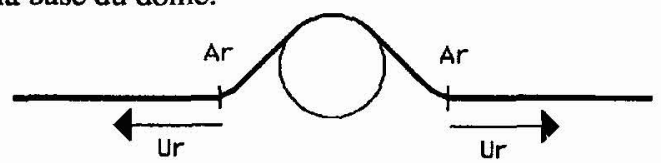

FIGURE 3 : Vitesse de relèvement de la plaque

Si l'on augmente la vitesse du projectile, on constate que le dôme ne peut s'agrandir et il y a perforation de la plaque. D'autre part, la surface délaminée diminue. Il semble, donc, que le délaminage, s'il peut se développer, absorbe une quantité d'énergie suffisante à l'arrêt du projectile. C'est pourquoi, un modèle, dans lequel l'énergie consommée par délaminage est calculée, a été développé. 


\section{IV - MODELISATION ENERGETIQUE}

Dans ce modèle [13], les différents modes d'absorption d'énergie sont comptabilisés, puis déduits de l'énergie incidente du projectile pour en calculer sa vitesse à chaque instant.

\section{1 - PRESENTATIONDUMODELE}

Les hypothèses retenues sont les suivantes :

- le matériau est élastique fragile endommageable

- le projectile est indéformable

- le délaminage est le seul processus d'absorption d'énergie

\section{Principe :}

Le délaminage se propage dans le plan de la plaque à la vitesse $\mathrm{U}(\mathrm{t})$. Il est représenté par une grandeur scalaire notée $\mathrm{A}$. Les parties déjà perforées de la plaque ne se délaminent plus.

On démontre [13] que l'évolution de la vitesse du projectile est liée à l'extension du délaminage et à l'état de perforation par la relation:

$$
\dot{V}=-\Pi \frac{L h}{m V^{2}} U\left(\Sigma G_{i d}\right) \sqrt{A} \dot{p}
$$

avec:

$\mathrm{L}$ : longueur de la plaque $\mathrm{U}$ : vitesse d'extension du délaminage

$\mathbf{h}$ : épaisseur de la plaque $\mathrm{A}$ : variable de délaminage

$m$ : masse du projectile $p:$ perforation $(0 \leq p \leq 1)$

$\mathrm{V}$ : vitesse du projectile Gic: taux de restitution d'énergie critique de linterface $i$

A partir d'un état initial connu, $\mathrm{A}=0, \mathrm{~V}=\mathrm{Vi}, \mathrm{p}=0$, la vitesse du projectile et la quantité d'énergie absorbée par délaminage peuvent être calculées pour chaque état de perforation. Le calcul de $V(t)$ et $A(t)$ nécessite la connaissance des caractéristiques géométriques de la plaque, la vitesse $U$ et les taux de restitution d'énergie associés à chaque interface.

\section{Résultat:}

Une comparaison calcul-expérience portant sur la vitesse résiduelle permet, pour un matériau donné, de calculer le taux de restituttion d'énergie critique Gc (on considère que Gc est identique pour chaque interface). Ensuite, la vitesse résiduelle pour toute vitesse incidente peut être prédite. Une courbe de comparaison est présentée en figure 4. Le modèle est en accord avec les résultats expérimentaux, sauf pour le système T300/5208 où la vitesse limite de perforation calculée est trop importante.

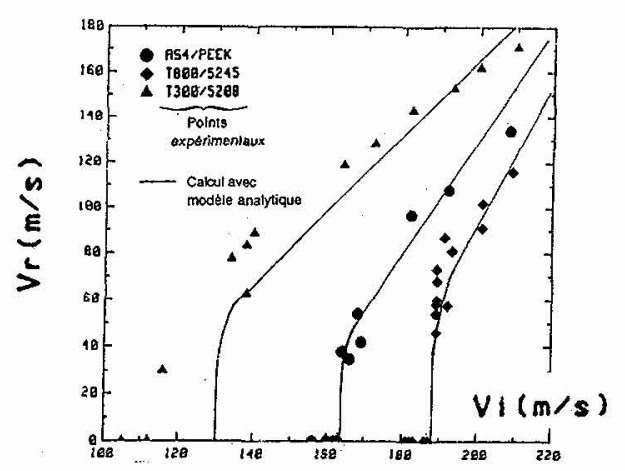

FIGURE 4 : Vitesse résiduelle en fonction de la vitesse incidente - Comparaison calcul/expérience

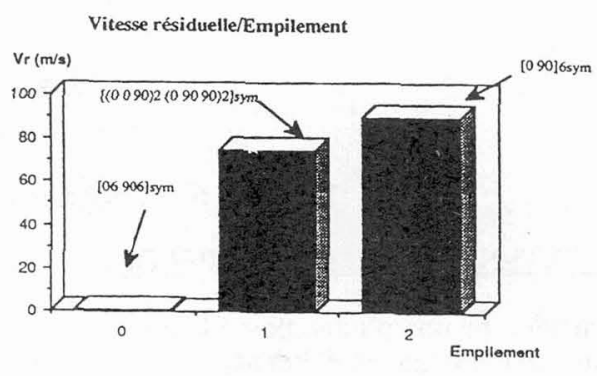

FIGURE 5 - Comparaison de l'énergie absorbée de plusieurs empilements

\section{2 -DISCUSSION: limites du modèle}

Le modèle ne permet pas de prendre en compte les effets d'empilement (figure 5).

On constate qu'il y a un mauvais accord sur les surfaces délaminées calculées et expérimentales. Ceci semble remettre en cause l'hypothèse selon laquelle le délaminage se propage pendant toute la durée du contact bille-plaque. 
L'approche énergétique ne permet d'accéder qu'à des taux de restitution d'énergie globaux. Il n'est pris en compte ni les modes de propagation du délaminage, ni les phases de la propagation.

\section{V - MECANISMES DE DELAMINAGE}

L'approche énergétique ne permet d'accéder qu'à des grandeurs globales (vitesse du projectile, énergie de délaminage, ....). L'approche ci-dessous précise certains paramètres.

\section{1-APPROCHE ADOPTEE}

L'endommagement pendant l'impact peut se schématiser par la succession de trois états :

- L'initiation du délaminage, correspondant à la phase où les défauts aux inter-plis apparaissent.

- La propagation du délaminage pendant laquelle le délaminage s'accroît dans le plan de la plaque.

- La dernière phase correspondant à l'arrêt de la propagation du délaminage.

Seule la phase de propagation sera étudiée ici. Celle-ci est gouvernée par la flexion de la plaque. En effet, des essais sur des plaques fixées en face arrière à un support indéformable montrent que la zone délaminée est nettement plus faible (figure 6).

\section{A - PLAQUE ENCASTREE AUX EXTREMITES}
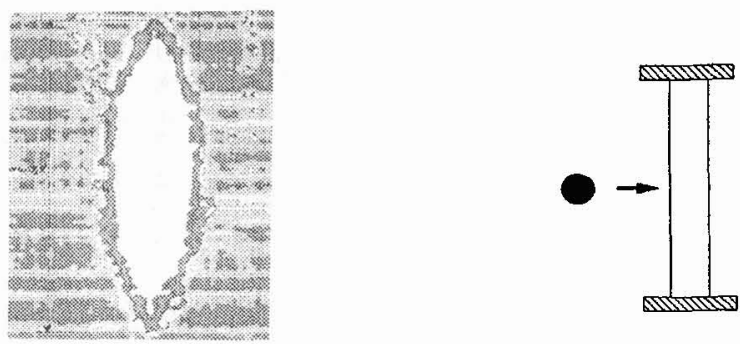

\section{B - PLAQUE CONFINEE EN FACE ARRIERE}

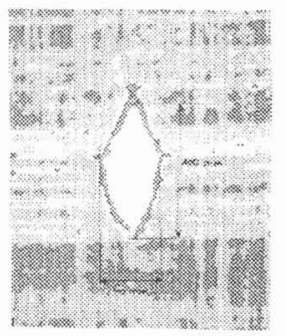

FIGURE 6 : Cartographies ultra-sonores de plaques impactées

\section{2 - EXTENSION DU DELAMINAGE}

Considérons une plaque possédant une fissure de délaminage. Sa propagation sera étudiée par éléments finis. Le maillage, en éléments coques, représente une plaque $\left(0_{6}, 90_{6}\right)_{\mathrm{s}}\left(6\right.$ plis à $0^{\circ}$ puis 6 plis à $90^{\circ}$, les 12 autres plis étant symétriques par rapport au plan central). Un défaut de rayon $\mathrm{Rf}$ est inséré entre le pli dixhuit et le pli dix-neuf (figure 7). A chaque incrément de temps, les taux de restitution d'énergie en modes 1,2 et 3 , respectivement $G_{1}, G_{2}$ et $G_{3}$ seront déterminés par la méthode des fermetures de fissures [1415]. Le taux de restitution d'énergie total est la somme de ces trois valeurs.

Le fond de fissure étant un cercle, $G$ peut être calculé en différents points de la pointe de fissure. Ainsi, $G$ est une fonction de l'angle entre la direction de calcul et l'axe des fibres du pli de la face avant. G- $0^{\circ}$ correspond au taux de restitution d'énergie calculé dans la direction des fibres du premier pli et G- $90^{\circ}$ dans la direction perpendiculaire. 


\section{Discussion:}

Les courbes de $\mathrm{G}$ pour $\mathrm{Rf}=45 \mathrm{~mm}$ sont présentées en figure 8 . Les vibrations créées lors du choc ne permettent pas au défaut de se propager puisque $G$ reste quasiment nul, dans les premiers instants. Ensuite, à partir d'un temps $t 1, \mathrm{G}$ crôit jusqu'à une valeur maximale Gmax. Le temps $t 1$ correspond à l'instant où le fond de fissure est sollicité en flexion. Il varie linéairement avec la taille du défaut.

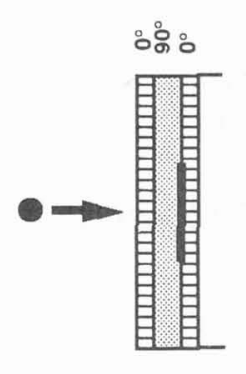

VUE DE PROFL FIGURE 7 VUE DE FACE

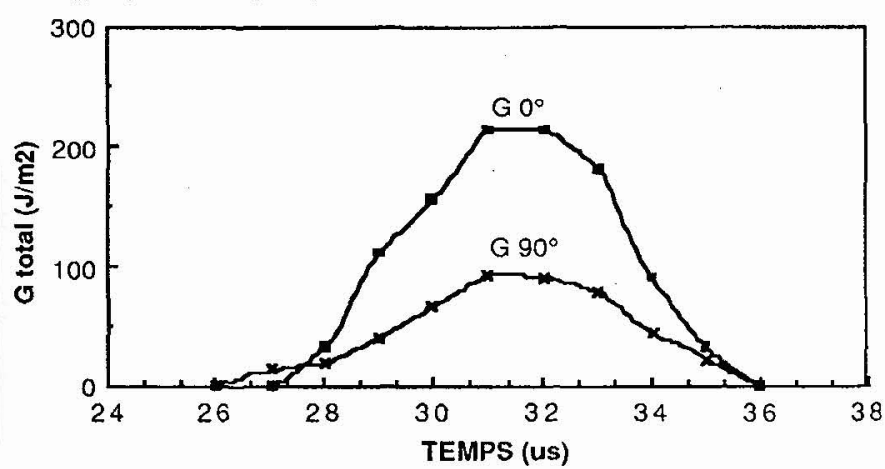

FIGURE 8 : $\mathrm{G}$ total calculé par éléments finis Défaut de rayon $45 \mathrm{~mm}$ - empilement $\left(0_{6}, 90_{6}\right) \mathrm{s}$

La courbe 8 montre une différence de taux de restitution d'énergie entre le sens fibre et le sens perpendiculaire. De même, expérimentalement, une forte anisotropie des dommages à l'interface considérée est constatée.

La comparaison de la déformée de la plaque et de la courbe $\mathrm{G}(\mathrm{t})$, montre que le relèvement (flexion) crée une ouverture locale du défaut. Cette ouverture suit le point de relèvement, et permet au délaminage de se propager. La méthode permet de calculer indépendamment les taux de restitution d'énergie dans les trois modes. Ainsi, leurs importances relatives peuvent être examinées. Dans notre cas, les taux de restitution d'énergie en mode 2 et 3 peuvent être considérés comme négligeables. C'est pourquoi $G \approx G_{1}$.

Un phénomène similaire a été visualisé sur des éprouvettes poutres unidirectionnelles. Ces éprouvettes sont préfissurées et la caméra ultra-rapide, permet d'observer en temps réel le fond de fissure. Lorsque le relèvement arrive au niveau du fond de fissure, il y a une ouverture très nette des deux lèvres de la fissure (figure 9).
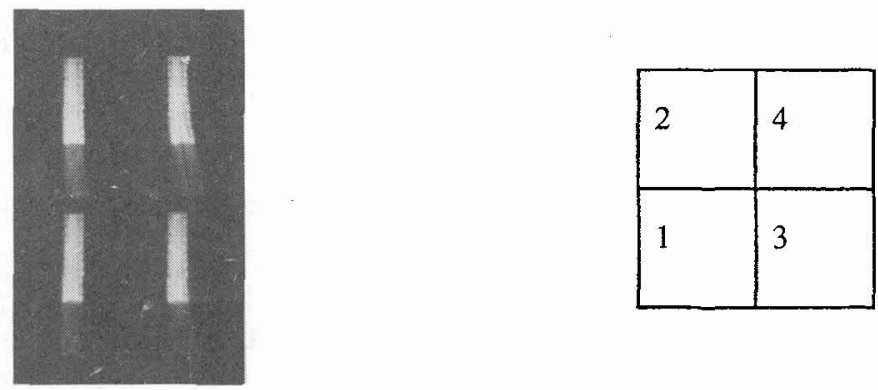

FIGURE 9 : Fond de fissure d'une poutre unidirectionnelle- 1 image par $20 \mu \mathrm{s}-\mathrm{Vi}=130 \mathrm{~m} / \mathrm{s}$

La valeur maximale de $\mathrm{G}$ dépend de la taille du défaut et de l'orientation par rapport aux fibres. Les taux de restitution d'énergie dans la direction $0^{\circ}$ et la direction $90^{\circ}$ sont très différents. Ceci est lié à l'anisotropie des caractéristiques homogénéisées en flexion des plis 1 à 18 et des plis 19 à 24 .

Après avoir caractérisé le mode de propagation du délaminage, nous allons maintenant nous intéresser à sa vitesse d'extension. 


\section{3 - VITESSE D'EXTENSION DU DELAMINAGE}

Expérimentalement, il a été mis en évidence que plus l'énergie de l'impact peut se délocaliser vite, plus la vitesse limite de perforation d'une plaque est élevée. Ceci montre bien qu'il est très important de pouvoir calculer la cinématique d'extension du délaminage. Comme il a déjà été dit, la vitesse U d'extension du délaminage n'est autre que la vitesse de relèvement Ur de la plaque. Une méthode de calcul de Ur est présentée puis comparé à la solution numérique.

\section{Méthode analytique:}

Analytiquement, le problème peut être posé de la manière suivante : considérons une plaque rectangulaire de côté $a$ et $b$ suivant les axes $x$ et $y$. Cette plaque est posée sur appuis simples aux extrémités $(x=0, x=a$, $y=0$ et $y=b)$. Une force transversale est appliquée en un point $M_{p}$ de la plaque. La déformée w $(x, y, t)$ vérifie l'équation $(1)$ :

$\rho h \frac{\partial^{2} w}{\partial t^{2}}+D_{11} \frac{\partial^{4} w}{\partial x^{4}}+4 D_{16} \frac{\partial^{4} w}{\partial x^{3} \partial y}+2\left(D_{12}+2 D_{66}\right) \frac{\partial^{4} w}{\partial x^{2} \partial y^{2}}+4 D_{26} \frac{\partial^{4} w}{\partial x \partial y^{3}}+D_{22} \frac{\partial^{4} w}{\partial y^{4}}=P(x, y, t)$

avec :

- les composantes de la matrice de rigidité en flexion: $\quad D_{i j}=\int_{-h / 2}^{h / 2} C_{i j} z^{2} d z$

- le chargement :

$\mathrm{P}(\mathrm{x}, \mathrm{y}, \mathrm{t})=\mathrm{p}_{0}(\mathrm{t}) \delta\left(\mathrm{x}-\mathrm{x}_{1}\right) \delta\left(\mathrm{y}-\mathrm{y}_{1}\right)$

- les conditions initiales :

à $\mathrm{t}=0: \mathrm{w}=\frac{\partial \mathrm{w}}{\partial \mathrm{t}}=0$

- les conditions aux limites :

$$
\left\{\begin{array}{l}
\text { en } x=0 \text { et } x=a: w=\frac{\partial^{2} w}{\partial x^{2}}=0 \\
\text { en } y=0 \text { et } y=b: w=\frac{\partial^{2} w}{\partial y^{2}}=0
\end{array}\right.
$$

Dans le cas d'un empilement orienté à 0 et à 90 degrés, $\mathrm{D}_{16}$ et $\mathrm{D}_{26}$ sont nuls. CHOW démontre [16] que la solution de ce problème, avec un chargement constant dans le temps, est :

avec :

$$
w(x, y, t)=\sum_{m=0}^{\infty} \sum_{n=0}^{\infty} \frac{a_{m n}}{\rho h b_{m n}} \sin \left(b_{m n} t\right) \sin \left(\frac{m \pi x}{a}\right) \sin \left(\frac{n \pi y}{b}\right)
$$

$a_{m n}=\frac{4 p_{o}}{a b} \sin \left(\frac{m \pi x}{a}\right) \sin \left(\frac{n \pi y}{b}\right) \quad$ et $\quad b_{m n}^{2}=\frac{\pi^{4}}{p h}\left[D_{11} \frac{m^{4}}{a}+2\left(D_{12}+2 D_{66}\right)\left(\frac{m n}{a b}\right)^{2}+D_{22}\left(\frac{n}{b}\right)\right]$

Le point de relèvement est le premier point de tangente horizontale (figure 3). La vitesse de relèvement est mesurée en déterminant le déplacement du point de relèvement pendant un intervalle de temps donné.

Nous allons maintenant présenter la méthode de détermination de Ur par éléments finis qui permettra de valider le calcul présenté ci-dessus.

\section{Méthode par éléments finis:}

Cette simulation représente une bille animée d'une vitesse Vi qui impacte une plaque isotrope. La vitesse Ur est déterminée à partir de la déformée de la plaque à différents instants, de la même manière que pour la méthode déja présentée.

Une mesure de Ur(t) au cours de l'impact a été effectuée. On constate que Ur atteint une valeur asymptotique après quelques trente micro-secondes (figure 10). 
D'autre part, le calcul numérique permet de montrer que la vitesse de relèvement de la plaque est indépendante de la vitesse incidente du projectile.

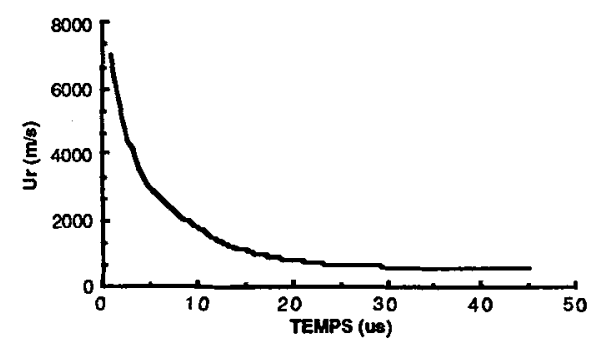

FIGURE 10 : Vitesse de relèvement au cours de l'impact

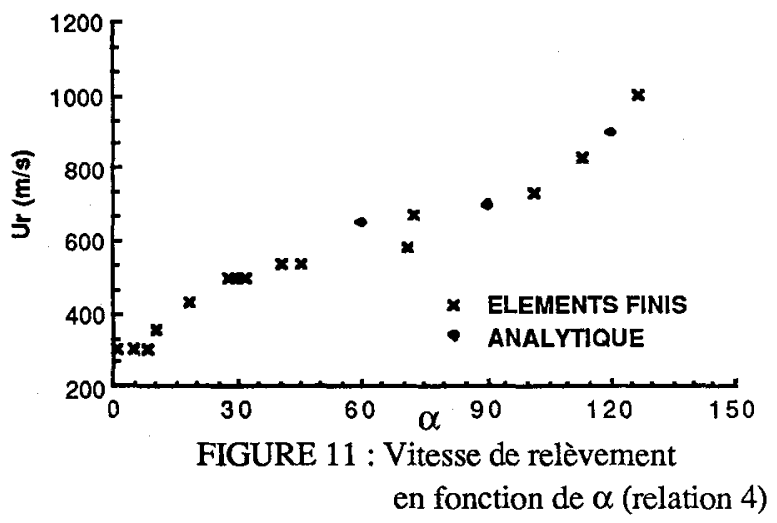

Comparaison des deux méthodes:

Les deux méthodes sont comparées dans le cas d'une plaque isotrope. L'équation du problème devient alors :

$$
\frac{\partial^{2} w}{\partial t^{2}}+\alpha\left(\frac{\partial^{4} w}{\partial x^{4}}+\frac{\partial^{4} w}{\partial x^{2} \partial y^{2}}+\frac{\partial^{4} w}{\partial y^{4}}\right)=P(x, y, t)
$$

avec :

$$
\alpha=\frac{E h^{2}}{12 \rho\left(1-v^{2}\right)}
$$

$\checkmark$ est le cœefficient de poisson

E est le module d'Young du matériau $\rho$ est la densité du matériau

$h$ est l'épaisseur de la plaque

Les seuls paramètres qui peuvent varier dans cette équation sont $E, h, \rho, v$ et la charge $P$. Les deux méthodes montrent que E,h,p,v ont une influence sur la vitesse $U r$. Par contre, Ur reste constante tant que $\alpha$ est constant. Si $\alpha$ varie, une évolution de Ur est constatée (figure 11).

\section{Discussion :}

Un bon accord est constaté entre les deux méthodes de calcul de Ur (figure 11). On peut, donc, considérer que les hypothèses faites dans la résolution analytique du problème sont correctes. Nous avons tracé les points de relèvement calculés analytiquement pour $\mathrm{t}=10,40,70,100 \mu \mathrm{s}$ (figure 12). Une relation entre le relèvement de la plaque et ses caractéristiques homogénéisées en flexion est mise en évidence.
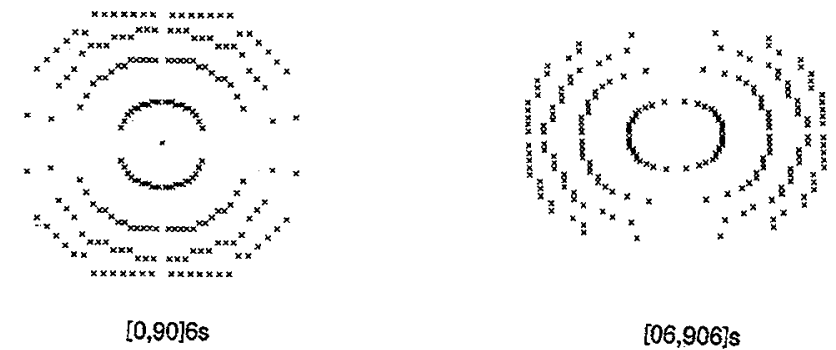

$[06,906] \mathrm{s}$

FIGURE 12 : Relèvement de plaques à $10,40,70,100 \mu$ s après le chargement 


\section{VI - CONCLUSION}

La modélisation énergétique de l'impact permet de prévoir avec une précision convenable, la vitesse résiduelle du projectile. Mais, cette approche très macroscopique ne permet pas de prévoir certains effets d'empilement. C'est pourquoi, une simulation par éléments finis a été utilisée.

Le calcul du taux de restitution d'énergie en fond de fissure d'une plaque impactée permet de mieux comprendre la propagation du délaminage. Lors du relèvement de la plaque, une ouverture locale qui va permettre au défaut de se propager est créée. Une forte différence de taux de restitution d'énergie est constatée entre la direction $0^{\circ}$ et la direction perpendiculaire dans le cas de l'empilement étudié. La cinématique de propagation du délaminage a été étudiée au travers de la vitesse de relèvement Ur. Les deux méthodes de calcul utilisées (éléments finis et analytique) donnent des résultats similaires. L'évolution du relèvement sur une plaque orthotrope a été calculée. On peut, ainsi, en déduire la surface délaminée à chaque instant de l'impact.

Il semble, maintenant, primordial de pouvoir comprendre comment le délaminage de la plaque cesse de s'étendre, alors que le dôme de flexion se propage jusqu'aux encastrements.

\section{REMERCIEMENTS}

Les auteurs remercient le Dr N. Beaumont pour son aide précieuse dans l'interprétation des résultats.

\section{REFERENCES}

/1/ Dorey G., Agard LS, 124, pp 6.1-6.11, 1982

/2/ Hong S.,Lu D., How to apply advanced composites technologies, pp 25-29,ASM INTERN, 1988

13/ Hong S., Liv D., Experimental mechanics, vol.29, $\mathrm{n}^{\circ}$ 2, pp 115-120, 1989

14/ Prichard J.C., Hogg P.J., Composites, Vol.21, nº, 1990

15/ Joshi S.P., Sun C.T., Second Technical Conference of the American Society for Composites, pp 177-185, 1987

16/ Joseph E., Grady, Sun C.T., American Society for Testing and Materials, ASTM STP 907, pp $5-31,1986$

17/ Grady J.E., Chamis C.C., Aiello R.A., American Society for Testing and Materials, NASA TM 100192, 1987

/8/ Grady J.E., Lewis Structures Technology, Vol.2 : Structural Mechanics, pp 2.235-2.244, 1988

19/ Sun C.T., Grady J.E., Composites Science and Technology 31, pp 55-72, 1988

/10/ Verpoest I., Li L., Doxsee L., Scholle M., Fourth European Conference on Composite Materials, pp 925-930, 1990

11/ Hsi-Yung T. Wu, George S. Springer, Journal of Composite Materials, Vol 22, pp 533-559, 1988

/12/ Liu D., Journal of Composite Materials, Vol. 22, pp 674-692, 1988

/13/ Beaumont N., Thèse Ecole Nationale Supérieure des Mines de Paris, Centre des Matériaux,18/12/1990

/14/ Jih C.J., Sun C.T., Engineering Fracture Mechanics, Vol.37, n 2, pp 313-322, 1990

/15/ Rybicki E.F., Kanninen M.F., Engineering Fracture Mechanics, pp 931-938, vol. 9, 1977

116/ Chow T.S., Journal of Composite Materials, pp 306-319,vol. 5, 1971 